\title{
Post-sternotomy pain syndrome following cardiac surgery: case report
}

\author{
Bruno Bordoni' \\ Fabiola Marelli2,3 \\ Bruno Morabito 2,3 \\ Beatrice Sacconi ${ }^{4,5}$ \\ Paolo Severino ${ }^{6}$ \\ 'Department of Cardiology, \\ Foundation Don Carlo Gnocchi, \\ IRCCS, Institute of Hospitalization \\ and Care, S Maria Nascente, Milan, \\ Italy; ${ }^{2}$ CRESO, School of Osteopathy \\ Centre for Research and Studies, \\ Castellanza, Italy; ${ }^{3}$ CRESO, School \\ of Osteopathy Centre for Research \\ and Studies, Falconara Marittima, \\ Italy; ${ }^{4}$ Department of Radiological, \\ Oncological and Anatomopathological \\ Sciences, Sapienza University of Rome, \\ Rome, Italy; ${ }^{5}$ Center for Life Nano \\ Science at Sapienza, Istituto Italiano di \\ Tecnologia, Rome, Italy; ${ }^{6}$ Department \\ of Cardiovascular, Respiratory, \\ Nephrology, Anesthesiology and \\ Geriatric Sciences, Sapienza \\ University of Rome, Rome, Italy
}

This article was published in the following Dove Press journal: Journal of Pain Research

15 May 2017

Number of times this article has been viewed
Abstract: Over 2 million people undergo sternotomy worldwide for heart surgery each year, and many develop post-sternotomy pain syndrome (PSPS) which persists in the anterior thorax. In some patients, PSPS lasts for many years or suddenly reappears a long time after the sternotomy. The exact etiology of PSPS is unknown. This article presents a case report of a patient with a diagnosis of PSPS (after cardiac surgery 4 years prior) for whom an osteopathic approach was used, which successfully eliminated the pain. In a previous study, we demonstrated that this osteopathic procedure could reduce sternal pain associated with a recent surgical wound. Further efforts are needed to understand the reasons for PSPS. In light of new scientific data, these osteopathic techniques could contribute to a multidisciplinary approach to solve the problem. Keywords: post-sternotomy pain syndrome, osteopathic, fascia, chronic pain, cardiac surgery

\section{Introduction}

Post-sternotomy pain syndrome (PSPS) is defined as discomfort after thoracic surgery, persisting for at least 2 months, and without apparent cause. ${ }^{1}$ It is estimated that over 2 million people worldwide undergo median sternotomy for heart surgery each year. ${ }^{1,2}$ In these patients, persistent thoracic pain is defined as PSPS, chronic post-sternotomy pain, or persistent post-sternotomy pain in the literature. ${ }^{1,3}$

PSPS is a common problem, with an incidence of $7 \%-66 \%$ within 1 or 2 years after the operation, in patients undergoing thoracic surgery. ${ }^{2,4-6}$ To be defined as PSPS, the pain must be continuously present up to 2 months post-intervention, and pre-existing causes which may lead to pain in the thorax should not be present. ${ }^{7}$ The exact etiology of PSPS is unknown. There are different reasons for these symptoms, and they do not seem to be related to the type of intervention. To our knowledge, the source of this pain has not been studied, and attempts have not been made to distinguish the contributions of different types of pain, such as somatic pain, visceral pain, and neuropathic pain or that resulting from the scar itself. ${ }^{6}$ In some patients PSPS can last for many years; in others it can suddenly reappear years after the sternotomy. ${ }^{4}$

In the past 30 years, numerous hypotheses have been considered, including entrapment neuropathy caused by sutures or scar tissue at the site of sternotomy, and intercostal neuralgia resulting from damage to the intercostal nerves during the dissection of the internal mammary artery. ${ }^{4,78}$ Moreover, it has been suggested that PSPS can be a result of rib fractures related to the surgical procedure, an injury to the brachial plexus due to patient positioning during the intervention, ${ }^{4}$ costo-sternal syndrome, or an allergy to metal sutures (nickel). ${ }^{4,5}$ Alternatively, chronic sternal pain may be related to structural changes
Correspondence: Bruno Bordoni Department of Cardiology, Foundation Don Carlo Gnocchi, IRCCS, Institute of Hospitalization and Care, S Maria Nascente, Via Capecelatro 66, Milan 20100 , Italy

Tel +39023496300617

Email bordonibruno@hotmail.com 
in the peripheral nerve endings, which would send altered afferent impulses to the spinal cord and the central nervous system, leading to allodynia. Psychological factors could contribute to this syndrome. Moreover, patients undergoing emergency sternotomy for heart surgery are at greater risk of chronic pain. ${ }^{2}$

Factors associated with age and gender may contribute to PSPS exacerbations. In particular, females younger than 70 years of age may be more likely to experience these pains. ${ }^{2,4,5}$ However, according to a large retrospective multicenter survey, older patients are more prone to this syndrome, indicating controversy regarding the effects of age. ${ }^{9}$ Other contributing factors are reported to include large waist circumference or obesity, smoking history, and preoperative depression or anxiety. ${ }^{5,10}$

Some authors state that this syndrome can also involve other areas of the body which are sore or stiff, in addition to the sternum. ${ }^{10,11}$ Currently, neither a specific therapy which is able to reduce the onset of the syndrome, nor a preferred surgical procedure to avoid PSPS, exist.

This article presents a case report of a patient with a diagnosis of PSPS after sternotomy for cardiac surgery 4 years prior, in whom osteopathic therapy led to the resolution of pain. This osteopathic intervention is a standardized procedure which we demonstrated to be successful in the reduction of sternal pain in a previous study of patients with recent surgical wounds (maximum 8 days after surgery). This therapy is only used in our center, at the Foundation Don Carlo Gnocchi, IRCCS (Istituto di Ricovero e Cura a Carattere Scientifico). In our previous study using this approach, we found that it reduced sternal pain, accelerated clinical stabilization, and facilitated earlier discharge. ${ }^{12}$

Osteopathic manipulative treatment (OMT) is defined as: "The therapeutic application of manually guided forces by osteopathic physician, to improve physiologic function and/or support homeostasis that has been altered by somatic dysfunction."13 The somatic dysfunction is defined as: "Impaired or altered function of related components of the somatic (body framework) system: skeletal, arthrodial and myofascial structures, and their related vascular, lymphatic, and neural elements." ${ }^{14}$

The clinical efficacy of OMT has been documented in different scenarios. OMT is effective in reducing migraine attacks. In a randomized controlled trial, OMT reduced chronic migraine pain, use of drugs, and functional disability in headache patients, compared with a placebo group. ${ }^{15}$

In a randomized, single-blind, placebo-controlled crossover study, OMT decreased shoulder pain intensity in breast cancer survivors, and increased range of motion to a greater degree than in the control group. ${ }^{16}$
A prospective, randomized, multicenter trial showed that OMT is effective in reducing length of stay in a population of preterm infants, without collateral effects. ${ }^{17}$

In another multicenter randomized controlled trial, OMT reduced the hospital mortality rate and length of stay in elderly patients with pneumonia. ${ }^{18}$ Several studies have shown that OMT reduces acute and chronic low-back pain, including in pregnant and post-partum women, more than expected placebo effects alone. Analgesic medication use is lower in OMT groups. ${ }^{19-22}$

\section{Case presentation}

A 67-year-old male had a sternotomy performed in 2012 for coronary artery bypass grafting (CABG), after presenting with an ST-segment elevation anterolateral myocardial infarction (STEMI). A triple CABG was performed by median sternotomy, with extracorporeal circulation and moderate hypothermia. A left internal mammary artery graft to left anterior descending coronary artery was utilized, and saphenous vein grafts from the aorta to the diagonal and obtuse marginal branches were performed. The sternum was closed using six stainless steel sutures and muscles, subcutaneous tissue and skin were sutured with absorbable synthetic woven threads. Neither infections nor sternal dehiscence were reported. Post-operative pain management included codeine (Hederix plan; Sit Laboratorio Farmac. Srl. Lombardy, Italy) (two tablets, $30 \mathrm{mg}$ ) and ibuprofen (Ibuprofene doc; Doc Generici Srl, Milan, Italy) (two tablets, $600 \mathrm{mg}$ ). The patient recuperated quickly in the hospital and was discharged after 5 days, and he suffered no complications. Rehabilitation in the hospital consisted of breathing exercises.

He was subsequently seen as an outpatient at the Department of Cardiology, Foundation Don Carlo Gnocchi, IRCCS. After surgery in 2012, the patient underwent a 3-week cardiac rehabilitation program at our department (2012), with free body exercises and progressive load on an exercise bicycle for 5 consecutive days per week.

When he presented to the clinic, his therapy included irbesartan (Irbesartan my: Mylan S.p.A, Milan, Italy) (150 mg), aspirin (Aspirinetta; Bayer S.p.A, Milan, Italy) (100 mg), clopidogrel (Clopidogrel teva; Teva Italia Srl, Milan, Italy) (75 mg), atorvastatin (Atorvastatina te; Teva Italia Srl) (20 mg), pantoprazole (Pantoprazolo zent; Zentiva Italia Srl) (20 mg), and pregabalin (Pregabalin teva ; Teva Italia Srl, Milan, Italy) (150 mg) daily. Physical examination showed regular heart rate and rhythm, no murmur, lungs clear to auscultation bilaterally with no rubs, rhonchi or wheezes; it also showed a soft, non-tender, non-distended abdomen 
with normal bowel sounds; no cyanosis, clubbing or edema in the extremities with the distal pulses present and equal. The patient had a normal weight, and was $165 \mathrm{~cm}$ tall with a waist circumference of $90 \mathrm{~cm}$, with no history of smoking or alcohol use. As to lifestyle, at that moment the patient was retired, not performing hard labor, and in good health with no chronic complaints. His electrocardiogram showed normal sinus rhythm of $60 \mathrm{bpm}$ and his blood pressure was 100/60 mmHg.

Echocardiography revealed mild concentric left ventricular hypertrophy and mild left ventricular dysfunction with an ejection fraction of $48 \%$, as assessed by Simpson's method, with first-degree diastolic dysfunction and no significant abnormality of the valve apparatus based on color-Doppler echocardiographic images.

The patient presented for evaluation of severe chest pain of cardiovascular origin, such as angina or other complications of the STEMI, had been excluded by his cardiologist. The patient had been started on pregabalin by his neurologist 3 months prior, for a presumed diagnosis of peripheral neuropathic pain. The pain mainly occurred during the patient's daily activities, when he was in an upright position. The pain made it impossible to rotate his head and neck to the right, but there was decreased pain intensity when he was in an extended position. The painful sensations were reported by the patient as feeling like a "belt tightening" in his sternum. His visual analog scale (VAS) pain assessment was performed

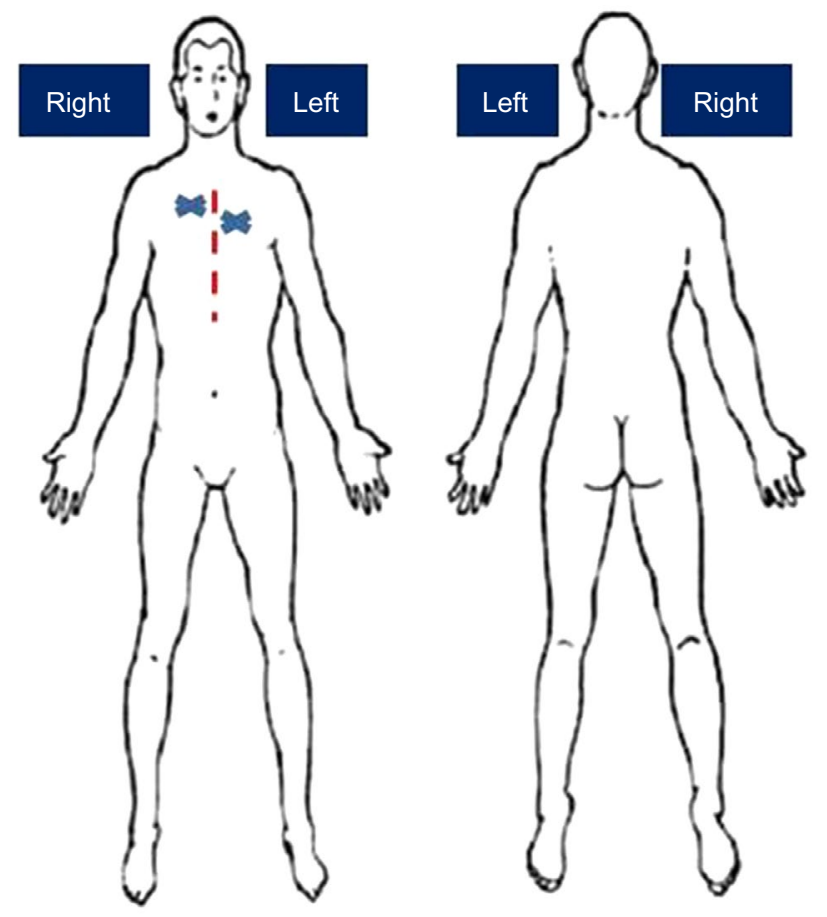

Figure I The area of the body affected by discomfort. by showing him a diagram of the human figure in its entirety, front and back, and he was asked to mark both the magnitude of the perceived pain and the area of the body affected by the discomfort (Figure 1). He reported a level of 7 on the VAS of pain intensity, centered in both the right side of the chest (between the lower clavicle and the fifth rib, without affecting the midline scar), and the left pericardial area (lower than the painful area of the right side, and without involving the scar). He was asked to lie down on a couch, slightly reclined with his legs stretched out, and with his chest accessible. A cardiologist examined him and confirmed the integrity of the sternum. His perceptions of the painful skin areas were evaluated, in order to determine whether any perceptual abnormalities were present.

To evaluate the skin surface responsiveness to external stimuli, a cotton pad was used, which allowed simultaneous evaluation of stimulus discrimination and epicritic sensitivity; sensitivity to pain was tested with a pin, and with slight pressure on different areas; thermal sensitivity was evaluated with a test tube containing hot water $\left(40^{\circ} \mathrm{C}-50^{\circ} \mathrm{C}\right)$ which was placed on the skin. During the skin assessment, regardless of the stimulus (cotton pad, pin, or hot water), the patient did not show any type of neurological impairment, and demonstrated normal sensitivity. No allodynia, hyperalgesia, hypoalgesia, or hypoesthesia were evident. The patient did not report any pain during the examination, and the intensity of each stimulus by the numeric rating scale (NRS) given by the patient was always 0 . Pain intensity is often measured in clinical and research settings using the 0 to 10 NRS. ${ }^{3}$ Following the visit, the cardiologist diagnosed the patient with PSPS.

The patient provided written informed consent for the publication of this case report and images.

\section{Osteopathic treatment}

After a discussion with the cardiologist, and based on our previous study of osteopathic treatment of post-sternotomy pain in heart surgery patients, a similar procedure was offered to the patient. In a change from our previous practice, ${ }^{12}$ given the lack of a recent sternum wound, the OMT was performed with the patient in a seated position and not supine in bed.

The OMT technique used was a fascial unwinding therapy, an approach defined as an indirect technique utilized by osteopaths, that respects the patient's tissues and causes no pain. This approach has been successfully used for other somatic complaints, such as non-specific lumbar back pain and non-specific neck pain. ${ }^{23-25}$

Osteopathic techniques are designed to release fascial restrictions, to mobilize tight ligaments and drain congested lymphatic channels; the purpose of these therapies is to alter 
the mechanical properties of fascial tissues, such as density, stiffness, and viscosity, so that the fascia can more readily adapt to physical stresses. ${ }^{26}$

The OMT does not induce any movement but allows the underlying tissue to express intrinsic movements, reflecting the physiological sliding of different fascial layers while breathing. Laying on the hands and following fascial vector directions, the application ends when the subcutaneous tissue becomes less rigid and when the movements expressed by the various palpated body segments are broader and similar to those of the (normal) contralateral side of the body. ${ }^{27}$

Osteopathic sessions were carried out over 5 consecutive days, with OMT applied for a maximum of 15 minutes. The OMT included three phases involving the whole mediastinum. During the first phase, the operator stands behind the patient, resting his hands on the thoracic outlet, with his index and middle fingers on the clavicle and the thumbs up to $\mathrm{C} 7$, facing each other, while the ring finger and little finger stay on the anterior area of the chest. In the second phase, the operator places one hand on the anterior area of the sternum, while the other hand is parallel on the patient's back; as the last phase, the hands are placed on the anterolateral area on the costal diaphragm (Figures 2-4).

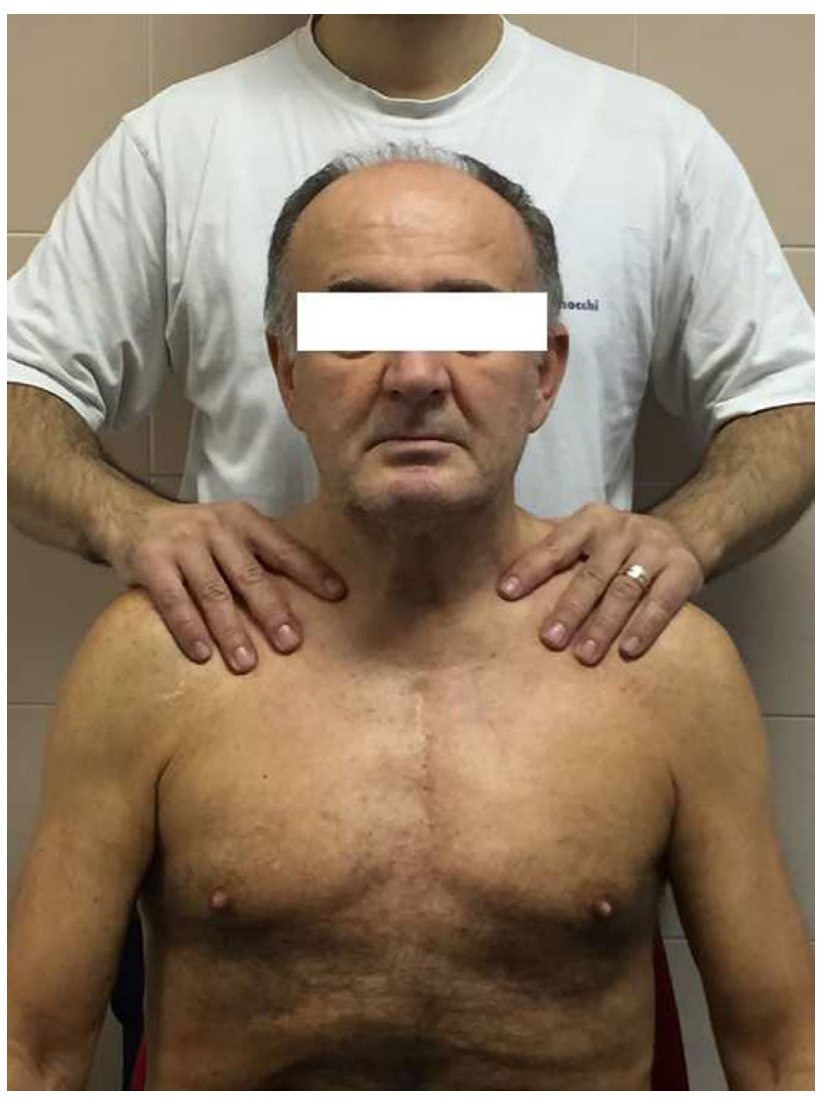

Figure 2 First phase of osteopathic manipulative treatment.

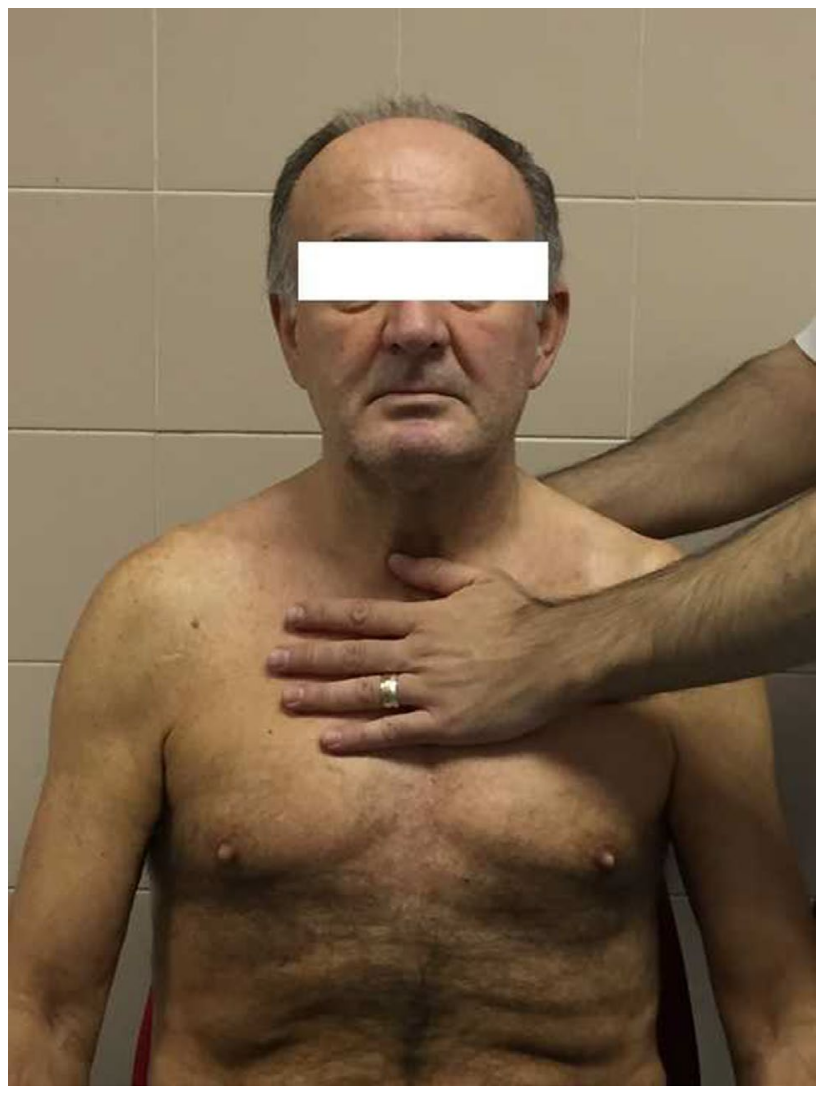

Figure 3 Second phase of osteopathic manipulative treatment.

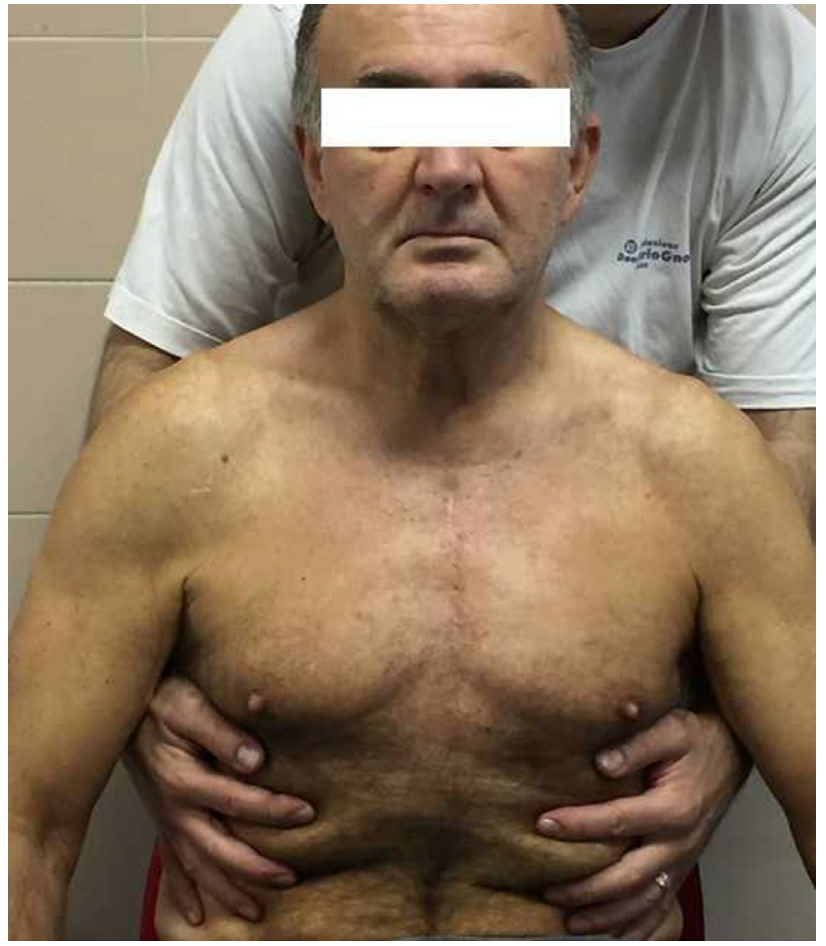

Figure 4 Third phase of osteopathic manipulative treatment.

During the day following the last OMT treatment, tests on the patient's skin surface were repeated, after a followup physical exam and findings of unchanged results by the 
cardiologist. At that visit, the patient's feeling of "belt tightening" was no longer present; moreover, head rotation in both directions did not produce any pain or difficulty with range of motion. On the VAS, the patient reported a pain level of 2. Remarkably, the patient was unable to give details of the most painful area, describing it as a minimal, widespread pain which changed location depending on daily physical activities, affirming that pain was no longer limiting movement; moreover, after the third session, the patient reported that pain did not wake him up during the night.

The treating neurologist tapered the dose of pregabalin, and when the patient was followed-up after 3 months to check his clinical status, there were no significant findings on physical examination, his cardiac status was stable, and he reported no abnormal cutaneous sensations. The VAS score was still reported to be 2. Pharmacological therapy was continued, except pregabalin, which had been discontinued. The patient's function was no longer limited, whereas widespread minimal pain persisted with no precise location on the body surface (Figure 5). Cervical movement no longer provoked pain.

The patient, having largely recovered, was given an appointment for a routine 1-year follow-up visit.

\section{Discussion}

This case report discusses a patient evaluated and successfully treated for PSPS with OMT, who developed his pain

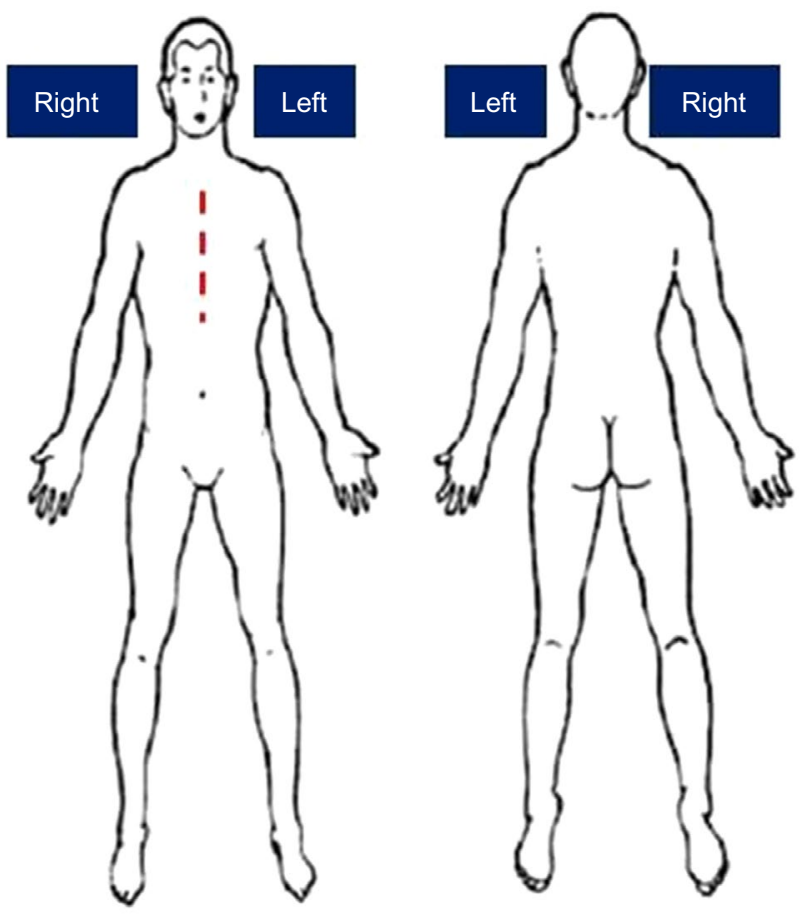

Figure 5 The absence of a precise location of pain. syndrome after a thoracotomy for CABG surgery 4 years prior. His presenting symptoms included severe pain in the anterior chest, and severe difficulty in completely rotating his neck to the right. After evaluation by a cardiologist to rule out active heart disease, the patient was diagnosed with PSPS. The patient took pregabalin, as prescribed by his neurologist, for neuropathic pain of peripheral origin.

Following the evaluation by the cardiologist, OMT fascial unwinding was performed with the patient seated, for a maximum of 15 minutes a day on 5 consecutive days.

At the end of these five osteopathic sessions, when the patient was seen for a follow-up visit after 3 months, the patient reported substantially diminished pain levels and restoration of normal neck movements in the absence of ongoing treatment with pregabalin.

At this time, many studies have shown that fascial osteopathic treatment is safe and efficacious for many clinical conditions, despite the fact that the exact somatic mechanisms by which fascial release works are not totally understood. ${ }^{26,27}$

In patients who have persistent anterior chest wall pain after a sternotomy for heart surgery, if an ongoing cardiac etiology is excluded, PSPS is a possible cause that is often not considered as a differential diagnosis. Chest pain may reduce a patient's quality of life, lead to sleep deprivation, delay return to normal activities of daily living, and cause adverse effects on cardiovascular and pulmonary functioning. ${ }^{12}$

One of the consequences of a sternotomy is the formation of adhesions in the scar tissue which form in the mediastinum, involving the somatic and visceral structures. ${ }^{28}$ All the retrosternal levels up to the pericardial sac may become involved in forming adhesions. During heart surgery, the pericardium is incised in order to perform surgical procedures, but it often leads to the formation of scar tissue at intrapericardial sites. ${ }^{29,30}$

These adhesions are not recognizable until new heart surgery is necessary, making the secondary procedure more complicated. ${ }^{31}$ These post-operative adhesions are usually vascularized and innervated. ${ }^{32}$

Specific data on the percentage of scar tissue formations and detection are not available. It is reasonable to assume that one of the causes underlying pain syndrome is the presence of these tissues following surgery, which begin to form within a few hours after the thoracotomy.

This was probably the case in this patient. Adhesions may have caused the pain, by altering mechanical forces in the mediastinum (because he only had pain when in the orthostatic position).

The fascial system, which gives rise to the adhesions, is rich in receptors. Mechanical forces may be detected in the 
adhesions by two types of receptors: the mechanoreceptor (or mechanosensor) and the mechanosensitive nociceptor. The first deals with information from mechanical modifications, whereas the second mostly generates information about painful stimuli. However, both convey somatic information. We can assume that in the presence of a skin scar or adhesion, these receptors may develop altered functions, which can result in transmission of non-physiological signals and creating pathological reflex arcs. ${ }^{33}$ Furthermore, the fascial system contains numerous receptors (Ruffini's and Pacinian corpuscles). If the microenvironment of the tissues in the fascia is altered by the presence of adhesions/scars, and the mechanical stimuli are no longer processed as normal physiological inputs, these receptors can become nociceptors and send afferent pain signals. ${ }^{34}$ The same fascial system contains its own nociceptors able to synthesize neuropeptides, which if present in altered mechanical structures, can produce an inflammatory environment and stimulate afferent signals related to pain. ${ }^{2}$

We can speculate that the analgesic capacity of OMT could be linked to an improvement of the mechanical stresses present in the mediastinum, improving the nociceptive afferents of this fascial continuum. ${ }^{12}$ Improved flexibility of the various fascial layers may allow "reset" of the afferent signals of the free nerve endings, resulting in physiologic response of the efferent ones.

Moreover, we know that OMT stimulates the parasympathetic system, decreasing the tone of the tissue and reducing the level of pro-inflammatory cytokines. ${ }^{35}$ We can assume that another mechanism for analgesic effects by OMT is related to the involvement of the parasympathetic system and an improved sliding of various fascial layers.

Another possible explanation for the reduction of pain by OMT is the placebo effect. We would have to design and execute trials with experimental OMT, control, and placebo groups to study this question about the efficacy of manual therapy in patients with PSPS.

The improvement of cervical movement may be related to the connection of the deep fascia of the neck to the endothoracic fascia, which covers the inner area of the chest and is in contact with the pericardium. The adhesions formed following thoracic surgery may have a greater percentage of myofibroblasts. The fibroblasts of the fascial system play a fundamental role in conveying tension and can dynamically affect mechanical tension. ${ }^{26}$ All fascial layers (from the skin to the pericardium) contain a variable amount of specialized fibroblasts (myofibroblasts), with the ability to contract. ${ }^{34}$ They contain a type of actin similar to that present in the smooth muscle of the digestive system; ie, alpha-smooth muscle actin. It is believed that in scar tissue and adhesions, these cells increase in number and as a result of the conditions present in the injured tissue, are stimulated to contract, consequently stimulating pathological symptoms. ${ }^{34,36-38}$

In this patient, we can assume that the mediastinal adhesions have caused new vectors of force which negatively influenced the movement of the neck. The OMT may have improved the force vectors, and influenced the position of the same connective tissue cells, to lead to more physiological behavior of the cells. ${ }^{39}$

OMT had no apparent side effects in this patient. In addition, the published literature on the fascial unwinding approach has not reported adverse effects resulting from this treatment.

\section{Conclusion}

It is estimated that each year over 2 million people worldwide undergo a sternotomy for cardiac surgery. Some of these patients develop persistent pain in the anterior chest which has been defined in literature as PSPS. The exact etiology of this pain is unknown.

The present article discussed a case report with pain resolution using OMT, in a patient who experienced PSPS after having a sternotomy for cardiac surgery 4 years prior.

We do not know the mechanisms of the analgesic effect seen after this approach. OMT may improve mechanical stress in the mediastinum and reduce the nociceptive afferent signals that develop following a surgical intervention, or could reduce the synthesis of inflammatory cytokines as well.

Further studies are needed to understand the basic mechanisms underlying PSPS. Further research will also be needed before we can regularly implement osteopathic therapies for chronic sternal pain.

\section{Disclosure}

The authors report no conflicts of interest in this work.

\section{References}

1. Costa MA, Trentini CA, Schafranski MD, Pipino O, Gomes RZ, Reis ES. Factors associated with the development of chronic post-sternotomy pain: a case-control study. Braz J Cardiovasc Surg. 2015;30(5):552-556.

2. van Gulik L, Janssen LI, Ahlers SJ, et al. Risk factors for chronic thoracic pain after cardiac surgery via sternotomy. Eur J Cardiothorac Surg. 2011;40(6):1309-1313.

3. Kalliomäki ML, Puolakka P, Huhtala H, Sisto T, Järvelä K. Bedside diagnosis of persistent post-sternotomy pain. Acta Anaesthesiol Scand. 2016;60(7):969-976.

4. Kalso E, Mennander S, Tasmuth T, Nilsson E. Chronic post-sternotomy pain. Acta Anaesthesiol Scand. 2001;45(8):935-939.

5. Rashidi S, Elenbaas TW, Hamad MA, van Suijlekom HJ, van Straten $\mathrm{AH}$. Does removal of steel wires relieve post-sternotomy pain after cardiac surgery? Asian Cardiovasc Thorac Ann. 2013;21(4):409-413. 
6. Lahtinen P, Kokki H, Hynynen M. Pain after cardiac surgery: a prospective cohort study of 1-year incidence and intensity. Anesthesiology. 2006;105(4):794-800.

7. Bruce J, Drury N, Poobalan AS, Jeffrey RR, Smith WC, Chambers WA. The prevalence of chronic chest and leg pain following cardiac surgery: a historical cohort study. Pain. 2003;104(1-2):265-273.

8. Dualé C, Ouchchane L, Schoeffler P; EDONIS Investigating Group, Dubray C. Neuropathic aspects of persistent postsurgical pain: a French multicenter survey with a 6-month prospective follow-up. J Pain. 2014;15(1):24.e1-24.e20.

9. Marcassa C, Faggiano P, Greco C, Ambrosetti M, Temporelli PL; Italian Association of Cardiovascular Prevention, Rehabilitation (GICRIACPR). A retrospective multicenter study on long-term prevalence of chronic pain after cardiac surgery. J Cardiovasc Med (Hagerstown). 2015;16(11):768-774.

10. Setälä P, Kalliomäki ML, Järvelä K, Huhtala H, Sisto T, Puolakka P. Postoperative hyperalgesia does not predict persistent post-sternotomy pain; observational study based on clinical examination. Acta Anaesthesiol Scand. 2016;60(4):520-528.

11. van Leersum NJ, van Leersum RL, Verwey HF, Klautz RJ. Pain symptoms accompanying chronic poststernotomy pain: a pilot study. Pain Med. 2010;11(11):1628-1634.

12. Racca V, Bordoni B, Castiglione P, Modica M, Ferratini M. Osteopathic manipulative treatment improves heart surgery outcomes: a randomized controlled trial. Ann Thorac Surg. Epub 2016 Jan 18.

13. American Osteopathic Association Guidelines for Osteopathic Manipulative Treatment (OMT) for Patients With Low Back Pain. JAm Osteopath Assoc. 2016;116(8):536-549.

14. Task Force on the Low Back Pain Clinical Practice Guidelines. American osteopathic association guidelines for osteopathic manipulative treatment (OMT) for patients with low back pain. J Am Osteopath Assoc. 2016;116(8):536-549.

15. Cerritelli F, Ginevri L, Messi G, et al. Clinical effectiveness of osteopathic treatment in chronic migraine: 3 -armed randomized controlled trial. Complement Ther Med. 2015;23(2):149-156.

16. Castro-Martín E, Ortiz-Comino L, Gallart-Aragón T, Esteban-Moreno B, Arroyo-Morales M, Galiano-Castillo N. Myofascial induction effects on neck-shoulder pain in breast cancer survivors: randomized, singleblind, placebo-controlled crossover design. Arch Phys Med Rehabil. 2016:S0003-9993(16)31313-2.

17. Cerritelli F, Pizzolorusso G, Renzetti C, et al. A multicenter, randomized, controlled trial of osteopathic manipulative treatment on preterms. PLoS One. 2015;10(5): 0127370.

18. Noll DR, Degenhardt BF, Johnson JC. Multicenter osteopathic pneumonia study in the elderly: subgroup analysis on hospital length of stay, ventilator-dependent respiratory failure rate, and in-hospital mortality rate. J Am Osteopath Assoc. 2016;116(9):574-587.

19. Licciardone JC, King HH, Hensel KL, Williams DG. OSTEOPAThic Health outcomes in chronic low back pain: The OSTEOPATHIC Trial. Osteopath Med Prim Care. 2008;2:5.

20. Schwerla F, Rother K, Rother D, Ruetz M, Resch KL. Osteopathic manipulative therapy in women with postpartum low back pain and disability: a pragmatic randomized controlled trial. J Am Osteopath Assoc. 2015;115(7):416-425.
21. Hastings V, McCallister AM, Curtis SA, Valant RJ, Yao S. Efficacy of osteopathic manipulative treatment for management of postpartum pain. J Am Osteopath Assoc. 2016;116(8):502-519.

22. Prinsen JK, Hensel KL, Snow RJ. OMT associated with reduced analgesic prescribing and fewer missed work days in patients with low back pain: an observational study. JAm Osteopath Assoc. 2014;114(2):90-98.

23. Minasny B. Understanding the process of fascial unwinding. Int JTher Massage Bodywork. 2009;2(3):10-17.

24. Tozzi P, Bongiorno D, Vitturini C. Fascial release effects on patients with non-specific cervical or lumbar pain. J Bodyw Mov Ther. 2011; 15(4):405-416.

25. Tozzi P, Bongiorno D, Vitturini C. Low back pain and kidney mobility: local osteopathic fascial manipulation decreases pain perception and improves renal mobility. J Bodyw Mov Ther. 2012;16(3):381-391.

26. Bordoni B, Zanier E. Understanding fibroblasts in order to comprehend the osteopathic treatment of the fascia. Evid Based Complement Alternat Med. 2015;2015:860934.

27. Schleip R, Findley TW, Chaitow L. Huijing PA. Fascia: The Tensional Network of the Human Body. London: Churchill Livingstone; 2012.

28. Alizzi AM, Summers P, Boon VH, et al. Reduction of post-surgical pericardial adhesions using a pig model. Heart Lung Circ. 2012;21(1):22-29.

29. Unsworth B, Casula RP, Kyriacou AA, et al. The right ventricular annular velocity reduction caused by coronary artery bypass graft surgery occurs at the moment of pericardial incision. Am Heart J. 2010;159(2): 314-322.

30. Cannata A, Petrella D, Russo CF, Bruschi G, Fratto P, Gambacorta M, Martinelli L. Postsurgical intrapericardial adhesions: mechanisms of formation and prevention. Ann Thorac Surg. 2013;95(5):1818-1826.

31. Orhan A, Görmüş N, Toy H, Görmüş IS, Çağlayan O, Tanyeli Ö. Prevention of retrosternal pericardial adhesions after cardiac surgery with mitomycin C. Heart Lung Circ. 2014;23(4):357-362.

32. Bove GM, Chapelle SL. Visceral mobilization can lyse and prevent peritoneal adhesions in a rat model. J Bodyw Mov Ther. 2012;16(1):76-82.

33. Bordoni B, Zanier E. Skin, fascias, and scars: symptoms and systemic connections. J Multidiscip Healthc. 2013;7:11-24.

34. Bordoni B, Zanier E. Clinical and symptomatological reflections: the fascia system. J Multidiscip Healthc. 2014;7:401-411.

35. Bordoni B, Marelli F, Morabito B, Sacconi B. Osteopathic treatment in a patient with left-ventricular assistant device with left brachialgia: a case report. Int Med Case Rep J. 2017;10:19-23.

36. Hoppe K, Schleip R, Lehmann-Horn F, Jäger H, Klingler W. Contractile elements in muscular fascial tissue - implications for invitro contracture testing for malignant hyperthermia. Anaesthesia. 2014;69(9):1002-1008.

37. Doe C, Bentley R, Behm DJ, et al. Novel Rho kinase inhibitors with anti-inflammatory and vasodilatory activities. J Pharmacol Exp Ther. 2007;320(1):89-98.

38. Tomasek JJ, Vaughan MB, Kropp BP, Gabbiani G, Martin MD, Haaksma CJ, Hinz B. Contraction of myofibroblasts in granulation tissue is dependent on Rho/Rho kinase/myosin light chain phosphatase activity. Wound Repair Regen. 2006;14(3):313-320.

39. Zein-Hammoud M, Standley PR. Modeled osteopathic manipulative treatments: a review of their in vitro effects on fibroblast tissue preparations. J Am Osteopath Assoc. 2015;115(8):490-502.

\section{Journal of Pain Research}

\section{Publish your work in this journal}

The Journal of Pain Research is an international, peer reviewed, open access, online journal that welcomes laboratory and clinical findings in the fields of pain research and the prevention and management of pain. Original research, reviews, symposium reports, hypothesis formation and commentaries are all considered for publication.
The manuscript management system is completely online and includes a very quick and fair peer-review system, which is all easy to use. Visit http://www.dovepress.com/testimonials.php to read real quotes from published authors. 